\title{
Standards for achieving a comfortable design for the Alzheimer's patients pathways
}

\author{
Radwan Alshawaf ${ }^{1}$ [0000-0002-2596-0353], and Ivan Krasnobaev ${ }^{1 *}$ [0000-0002-8401-4906] $^{2}$ \\ ${ }^{1}$ Kazan State University of Architecture and Engineering, 420043, Zelenaya St., Kazan, Russia
}

\begin{abstract}
The elderly is an essential part of society because of their interaction with their surrounding environment and their personal experiences. They were categorized into several levels based on the physiological and psychological needs. Alzheimer's patients constitute a good percentage within society, but there is no accurate data. In cases where the patient suffers from a progressive lack of spatial awareness, studies have developed several criteria to study semi-open spaces intended for the elderly. However, it did not set design standards. Consequently, this paper examines the design standards and requirements for Alzheimer's patients. It is a virtual practical study that simulates optimal standards.
\end{abstract}

Keywords. Alzheimer's disease, Alzheimer patients, planning, pathway design, pathway evaluation, Pathway criteria, Dementia, elderly, Architecture.

\section{Introduction}

As people are now living to an older age, it is inevitable that degenerative mental illness issues have become even more important. People who suffer from dementia diseases, the most important of which is Alzheimer's, is an important research topic, as studies focused on Alzheimer's are few compared to its importance and risk. The importance of the research lies in bridging the knowledge gap for the design standards for dementia patients. The research is focused on finding appropriate pathways according to the optimal standards to raise the comfort level for patients with Alzheimer's disease. It is necessary to take into account the psychological and physiological aspects of patients and providing an evaluation method for these pathways through which their quality and suitability for Alzheimer's patients can be measured.

Almost every nation in the world will gradually see an increase in the population of older adults. Between the years 2000 and 2050, the global population of people aged 85 and over is expected to rise at an unprecedented pace [1]. Since dementia affects $6 \%$ of all people aged 65 and older, the expected growth rate of the older population will also increase the burden of the disease [2,3]. According to the research, adopting more holistic medical approaches that offer therapeutic benefits through a range of interventions particularly those that integrate nature - it might be helpful for patients with Alzheimer's disease (AD) or dementia to improve their healthcare experience [4]. It is subsequently highly essential to develop age-friendly environments that are open, equitable, inclusive,

*Corresponding author: tpa.kgasu@gmail.com 
safe, and welcoming, while still maintaining synergy with the natural environment [5]. Studies on landscape have demonstrated the benefits of its various components $[6,7,8]$, such as green (grass fields or gardens) and blue (seas, lakes or rivers) [8,9] spaces, for individuals' physical well-being, especially for older adults [10]. It has been observed that elderly individuals have a correlation between higher exposures to sea views and lower levels of depression [9]. Further research has found that the therapeutic landscape's effect is primarily determined by the relationship or connection between people and their social environments based on their attitude, identity, and culture, and the fact that therapeutic for one person may be harmful to another [11]. This is why different parts of the globe have different ways of creating calming and therapeutic environments, not only for the general public, but also for the aged. For example, the use of Bonsai and scenic beauty in Japanese culture in contrast to the use of modern indoor recreational facilities in most western cultures. Researchers therefore advanced the idea to understand and analyze therapeutic effects of a broad variety of sites such as domestic environments as a result of the focus on location [12]. Due to the continuous expansion of the therapeutic landscape definition causing ambiguity, researchers suggest that the definition is to be divided into groupings categorized by different factors of importance to resolve this [13]. To further comfort increase and participation of people with dementia in everyday life, the creation of dementia-friendly interventions through many internal environmental improvements such as improved flooring, lighting, furniture, and visual signage were first implemented in indoor spaces of hospitals and long-term care facilities [14]. Since then, more improvements have being made in the planning and design of these therapeutic environments. The two main steps that should always be included in the optimal design process are environmental evaluation and site review [15]. Although, insights into the impact of environmental factors and assistive technologies are beneficial to design practice. The reviews discussed indicate many issues that hinder effective implementation of these insights in design practice. Though there are a few product design best practices that combine a variety of approaches with an emphasis on patient needs and technology These best practices are also heavily individualized, preventing generalizability to people with dementia in general. Furthermore, they typically focus on product (rather than environmental) design, and as a result, they focus on dyadic experiences between an individual with dementia and a specific caregiver or family member rather than several people with dementia (such as residents in care facilities). The best practices, on the other hand, may be extremely motivating [16]. A key factor in the improved care after people with dementia is the relationship between the patient and caregiver, with honesty, understanding and trust being the basis of the relationship. More research is required to understand how teaching affects understanding and trust when dealing with people who have dementia, according to the literature [17].

\section{Research methodology:}

The «pathways» are multi-functional. It is related to various, social, environmental, natural, and other design factors. Each of them has different methods of conducting studies and analysis, which are represented in the following aspects:

1-The theoretical part: Comparative method will be used according to the following:

-Comparative method: in diagnosing urban environment and its relationship in the digital simulation process, and comparing the theoretical side with the practical application; in terms of objectives, policies, types, levels, and stages of developing results; by collecting data and information from specialized references and sources.

2-The practical part: Quantitative method and the analytical synthetic method will be used. 
-Quantitative method: Emphasize objective measurements and the statistical, or numerical analysis manipulating pre-existing statistical data using computational techniques to extract standard tables that can be used as a reference for evaluation.

-The analytical synthetic method: involves drawing conclusions and formulating suggestions and recommendations when studying the table.

As a result, the integrated approach will be used in applied research, which is a summary of the previous curricula, serving the objectives of the study. As an applied scientific study, it draws from past experiences to create perceptions of the future. This allows the comprehensiveness to be exploited using the descriptive analytical synthetic method, and balance using the tools of quantitative analysis. It is based on the existence of a link between the scientific and theoretical framework of research and the applied and practical reality. This allows studying of all the factors and variables at the same time, which increases the possibility of generalizing the results and recommendations.

Using the information above, methodology generates the following steps:

- The literature review and deducing the appropriate standards for Alzheimer's patients in designing pathways

- presentation of these standards in a simplified table showing their divisions and facilitating their evaluation

- Digital simulation of appropriate external pathways for Alzheimer's patients reviewing architectural and medical literature and recommendations

- The application of the study to the Village Alzheimer ${ }^{1}$ project, as it contains studied paths that can be measured and evaluated using digital simulations

- Designing a virtual project based on the proposed standards in order to reach the best the application of the study to the Village Alzheimer project, as it contains studied paths that can be measured and evaluated using digital simulations

- outcome possible

- Discussing and submitting suggestions and recommendations

\section{Results and discussion}

\subsection{Pathways evaluation criteria}

Planners and architects mention the importance of the built environment, pathways of all kinds in planning and urban design, and this was indicated by Kevin Lynch ${ }^{2}$. He said that the image of the best environment gave a sense of reassurance, psychological comfort and self-realization and «a distinctive and legible environment not only offers security but also heightens the potential depth and intensity of human experience. Although life is far from impossible in the visual chaos of the modern city, the same daily action could take on new meaning if carried out in a more vivid setting» $[18,19]$.

The characterization of the pathway through the urban environment reflects a recent design process based on behavioral, sensory and physical criteria. Described in the literary references, and through the accurate description of the void of the urban atmosphere [2022]. It is possible to place the items of criteria for achieving the urban atmosphere within the pedestrian pathway in the residential villages of Alzheimer's patients. [23- 28] (Tables1, $2,3)$.

\footnotetext{
${ }^{1}$ Village Alzheimer It is a project designed by Nord Architects in Dax, France, which is a model village for Alzheimer's patients https://villagealzheimer.landes.fr

${ }^{2}$ Kevin Andrew Lynch an American urban planner and author. He is known for his work on the perceptual form of urban environments and was an early proponent of mental mapping
} 
Table 1. Pathway optical properties.

\begin{tabular}{|c|c|c|c|c|c|}
\hline \multicolumn{4}{|c|}{ Criteria } & & \\
\hline \multirow{14}{*}{ 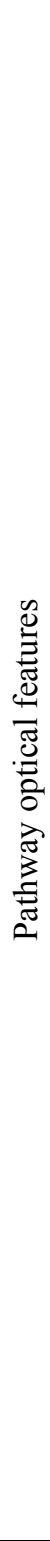 } & \multirow{10}{*}{ 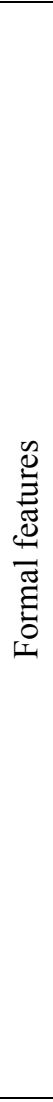 } & \multirow[t]{2}{*}{ In terms of shape } & $\begin{array}{c}\text { Presence of } \\
\text { shape }\end{array}$ & $\begin{array}{c}\text { Having a } \\
\text { distinctive shape } \\
(2 \text { marks })\end{array}$ & $\begin{array}{c}\text { Lack of } \\
\text { distinctive shape }\end{array}$ \\
\hline & & & Shape continuity & Continuous & $\begin{array}{l}\text { Intermittent } \\
\text { (1 mark })\end{array}$ \\
\hline & & \multirow[t]{2}{*}{$\begin{array}{l}\text { In terms of } \\
\text { contrast }\end{array}$} & $\begin{array}{l}\text { Presence of } \\
\text { antithesis }\end{array}$ & $\begin{array}{c}\text { The presence of } \\
\text { the distinct } \\
\text { contrast } \\
(1 \text { mark })\end{array}$ & $\begin{array}{l}\text { Lack of distinct } \\
\text { contrast }\end{array}$ \\
\hline & & & $\begin{array}{c}\text { Continuity of } \\
\text { contrast }\end{array}$ & Continuous & $\begin{array}{c}\text { Intermittent } \\
(1 \text { mark })\end{array}$ \\
\hline & & \multirow{2}{*}{$\begin{array}{l}\text { In terms of } \\
\text { redundancy }\end{array}$} & $\begin{array}{l}\text { The presence of } \\
\text { redundancy }\end{array}$ & $\begin{array}{c}\text { Presence of } \\
\text { distinct repetition }\end{array}$ & $\begin{array}{l}\text { Lack of distinct } \\
\text { repetition } \\
(2 \text { marks })\end{array}$ \\
\hline & & & $\begin{array}{c}\text { Continuity of } \\
\text { repetition }\end{array}$ & Continuous & $\begin{array}{c}\text { Intermittent } \\
(1 \text { mark })\end{array}$ \\
\hline & & \multirow[t]{2}{*}{$\begin{array}{l}\text { In terms of } \\
\text { texture }\end{array}$} & $\begin{array}{l}\text { Presence of } \\
\text { texture }\end{array}$ & $\begin{array}{c}\text { The presence of } \\
\text { the distinctive } \\
\text { texture } \\
(1 \text { mark })\end{array}$ & $\begin{array}{l}\text { Lack of distinct } \\
\text { texture }\end{array}$ \\
\hline & & & $\begin{array}{c}\text { Texture } \\
\text { continuity }\end{array}$ & Continuous & $\begin{array}{l}\text { Intermittent } \\
(1 \text { mark })\end{array}$ \\
\hline & & \multirow[t]{2}{*}{ In terms of color } & Presence of color & $\begin{array}{c}\text { The presence of } \\
\text { distinctive color } \\
\text { ( } 3 \text { marks })\end{array}$ & $\begin{array}{c}\text { Lack of } \\
\text { distinctive color }\end{array}$ \\
\hline & & & Color continuity & Continuous & $\begin{array}{c}\text { Intermittent } \\
(2 \text { marks })\end{array}$ \\
\hline & \multirow{4}{*}{ 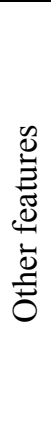 } & \multirow[t]{2}{*}{ In terms of smell } & $\begin{array}{l}\text { The presence of } \\
\text { odors }\end{array}$ & $\begin{array}{c}\text { The presence of } \\
\text { odors } \\
(2 \text { marks })\end{array}$ & $\begin{array}{c}\text { Lack of } \\
\text { persistent odors }\end{array}$ \\
\hline & & & Odor persistence & $\begin{array}{l}\text { Continuous } \\
(1 \text { mark })\end{array}$ & intermittent \\
\hline & & \multirow[t]{2}{*}{ In terms of sound } & $\begin{array}{l}\text { The presence of } \\
\text { sound }\end{array}$ & $\begin{array}{c}\text { The presence of } \\
\text { distinctive } \\
\text { sounds } \\
(2 \text { marks })\end{array}$ & $\begin{array}{l}\text { Lack of distinct } \\
\text { sounds }\end{array}$ \\
\hline & & & Sound continuity & $\begin{array}{c}\text { Continuous } \\
(1 \text { mark })\end{array}$ & intermittent \\
\hline
\end{tabular}


Table 2. Design characteristics of the pathways.

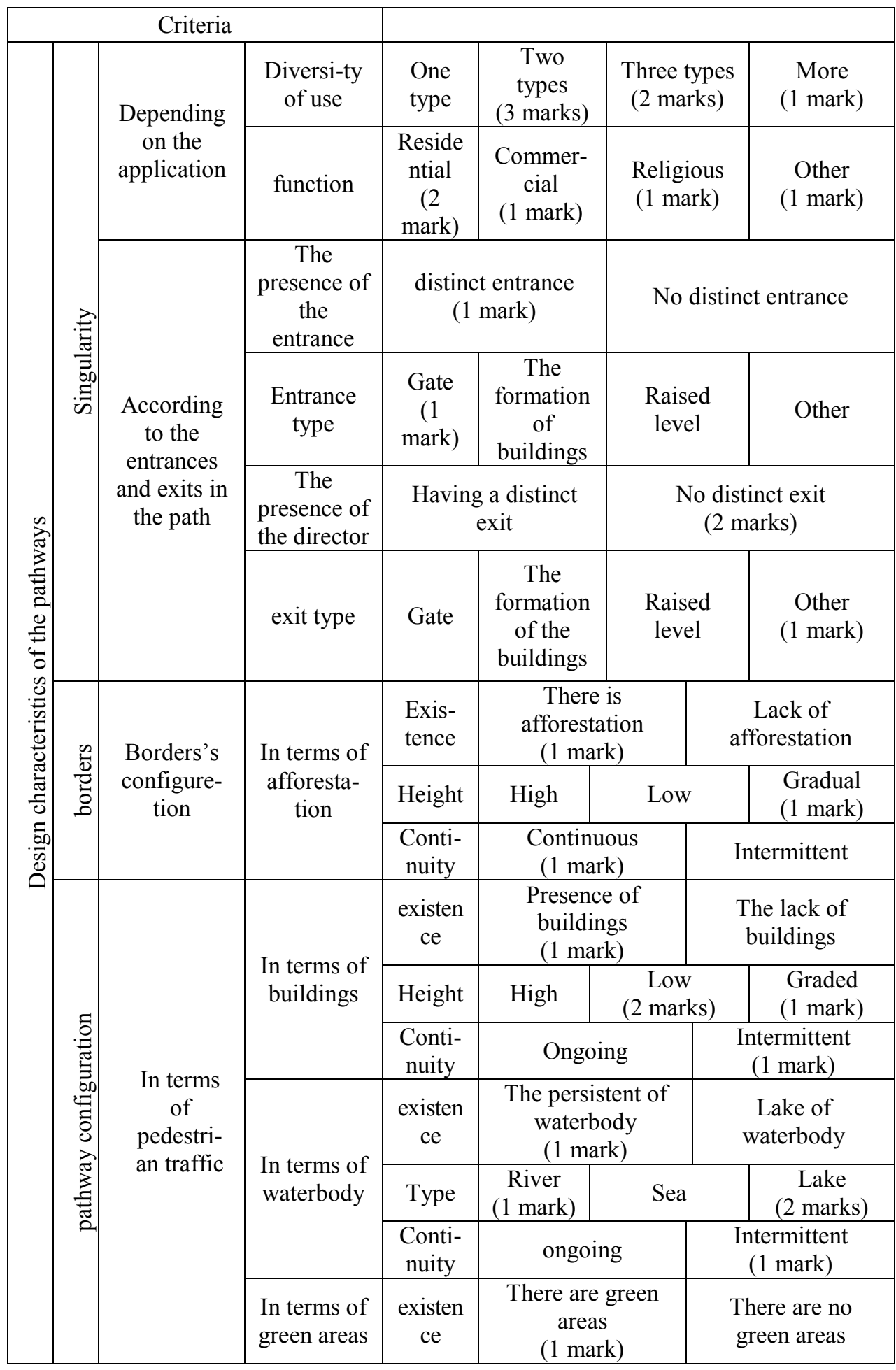




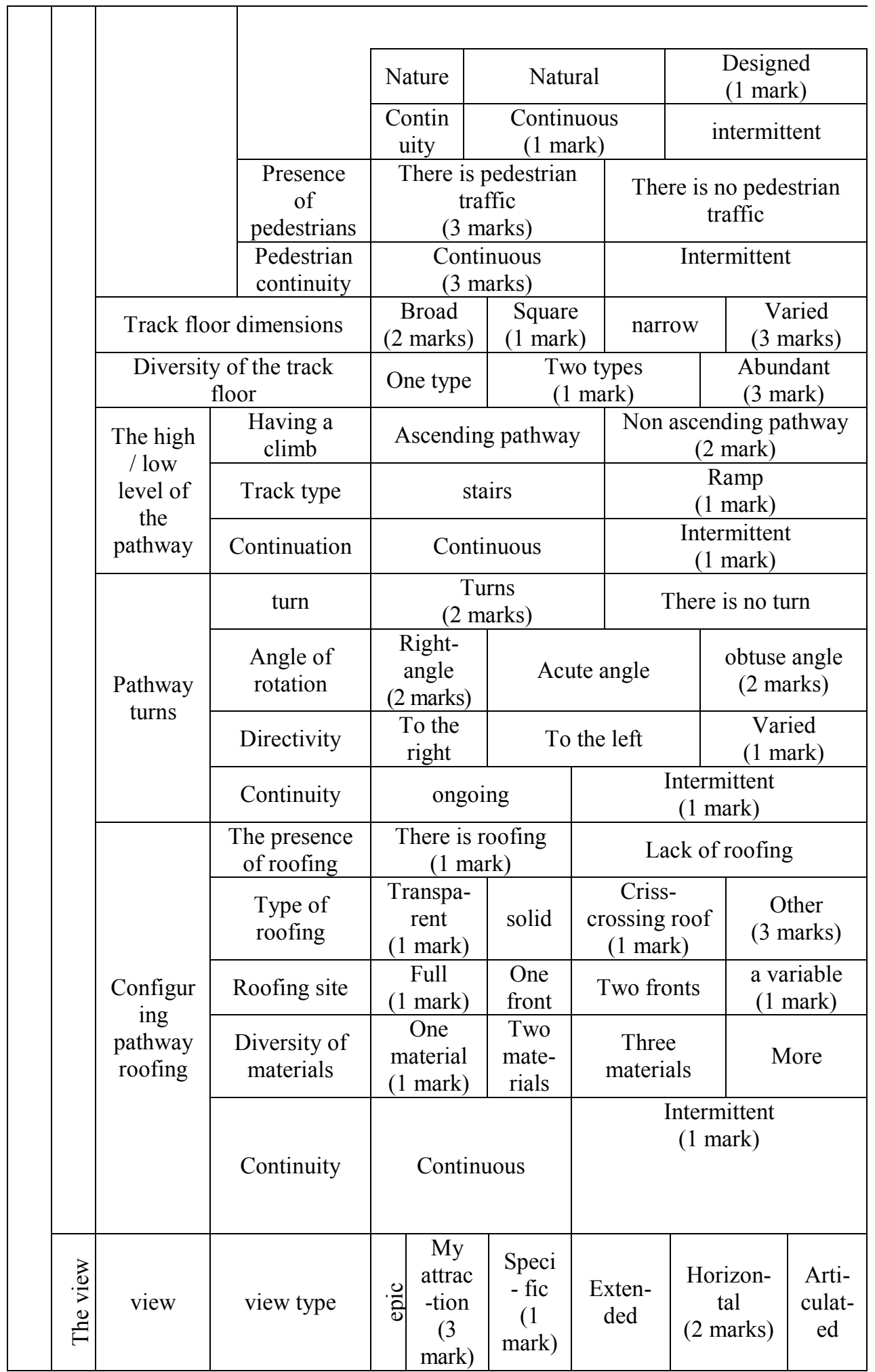




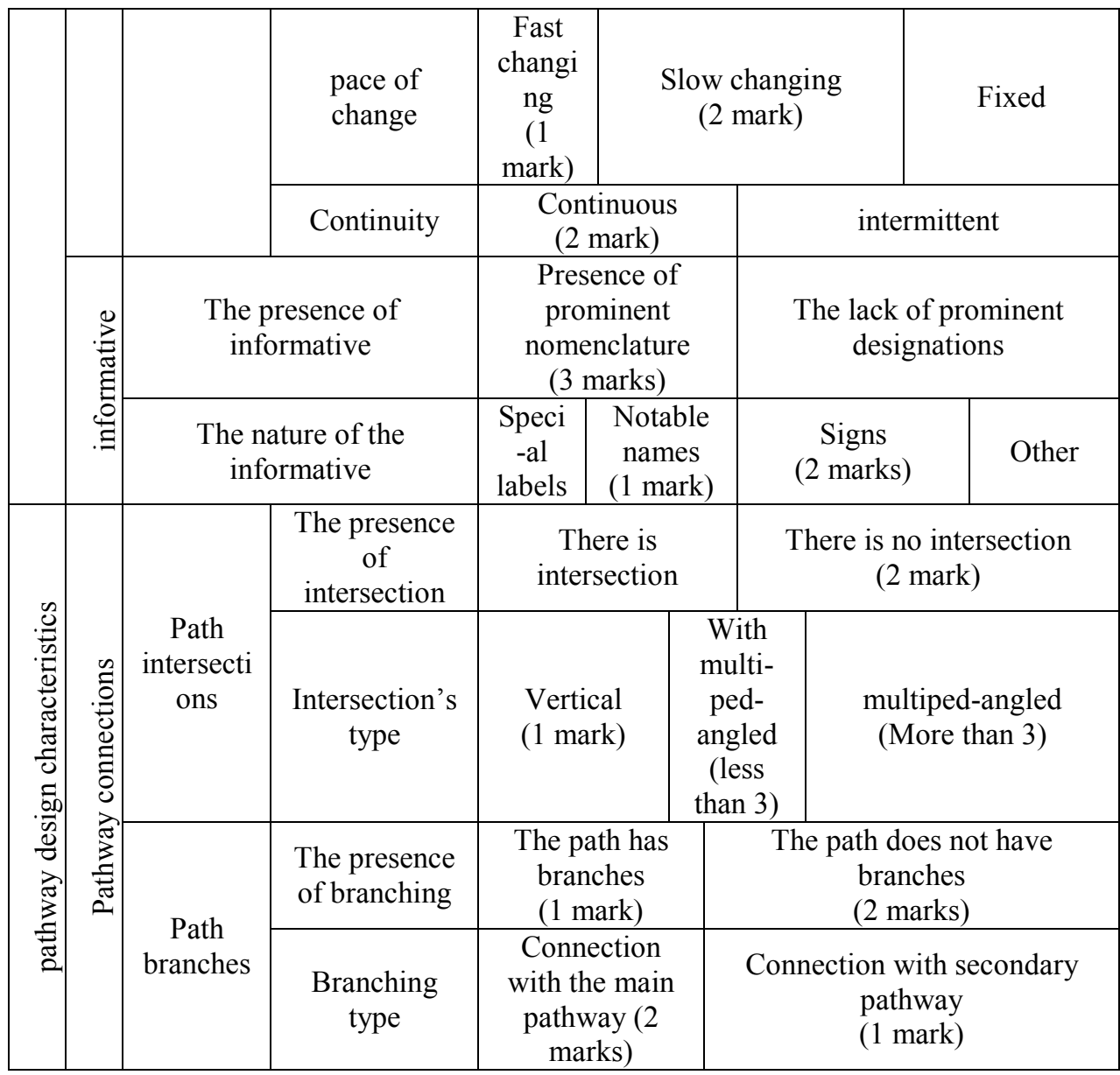

Table 3. Results evaluation.

\begin{tabular}{|c|c|c|}
\hline Percentage (\%) & Mark (from 80$)$ & Evaluation \\
\hline $60-69 \%$ & $48-55$ & Good \\
\hline $70-84 \%$ & $56-67$ & Very good \\
\hline $85-100 \%$ & $68-80$ & excellent \\
\hline
\end{tabular}

\subsection{Analysis of a similar project}

This digital simulation was applied to the paths in the Village Alzheimer ${ }^{1}$ project to evaluate and measure quality according to this study. Since the project received a (very good) evaluation from the researcher and was distinguished in terms of the visual characteristics of the pathways, we found that it had some distinctive shapes along the way. They had positive effects increasing the visual memory effectiveness for Alzheimer's patients, but it slightly lacked some color variation, as repeated use of the same time may not give the desired results.

\footnotetext{
${ }^{1}$ Village Alzheimer It is a project designed by Nord Architects in Dax, France, which is a model village for Alzheimer's patients https://villagealzheimer.landes.fr
} 
As for the appearance and design characteristics of the path, it was appropriate in terms of the diversity of the path's functions and types, and the entrances were clear and easy to find. However, the exits would have been better hidden by visual effects using trees and other natural methods than the technical and mechanical solutions. When it comes to green areas, it was distinguished by the fact that the designed natural environment was appropriate in terms of the classical ambiance suitable for the elderly and gradation in the size of the trees to control the visual factors appropriate for the patients. We can see the short trees crowded in some places and the long ones spaced out in order to increase the vision axis in the project's center, especially near the central lake of the path. Due to the project's focus on nature to a large extent, the issue of covering the pathways was neglected, for it was possible to add some pergolas to raise the efficiency of the project in summer at peak times

Summarily, the project paths are suitable for Alzheimer's patients. The attention is paid to signs and directional signals but there is an inappropriate increase in intersections that may cause some mental confusion for these patients. This may be as they use the closedloop method which gives the patients the option to go forward using points of interests like distinct building. This helps to reduce the number of options for the patients in order to help them choose a pathway.

\subsection{Case study according to standards}

Based on the previous study, a virtual project was designed according to these standards in order to present a clearer image regarding the most optimal pathways for Alzheimer patients taking into account the design and health recommendations (Fig. 1). Fig. 1 A presents a perspective that shows the pathways, the visual openness of the axes, and the linking of paths through interactive squares with different functions. Fig. $1 \mathrm{~B}$ is the site plan showing the pathways, their directions, their connection, and their relationship to the landscape. Fig. C proposed project section.

The digital simulation was applied to the paths of the proposed project, where it received an excellent grade according to the previous evaluation, where previous experiences were used and developed to obtain the best result possible. The optical features of the path were designed in a similar manner to the previous projects in terms of shapes and characteristics (contrast, repetition, texture, or others), but the color effects were taken into account in a more appropriate manner. A distinctive color was added to each area of the path using natural elements (trees blossoms, or others). As for the design characteristics, the entrances were emphasized through distinct gates for each building with attention to the idea of concealing the project exits through moving natural elements. The landscape, it was designed with appropriate visual elements such as trees, water elements and attractions that facilitate the decision-making process for Alzheimer's patients. Due to the topography of the available site, it was necessary to add slopes. Attention was paid to directing the pathways in order to influence the optical axes of Alzheimer's patients by designing the turns of paths and intersections so that the patient had a continuous vision of the closed loop, which did not prompt him/her to search for an exit. This leads to reducing the options for patients and thus reducing the severity of confusion because he/she still sees a way he one walk in, and this leads to reducing the options for patients and the severity of confusion. 


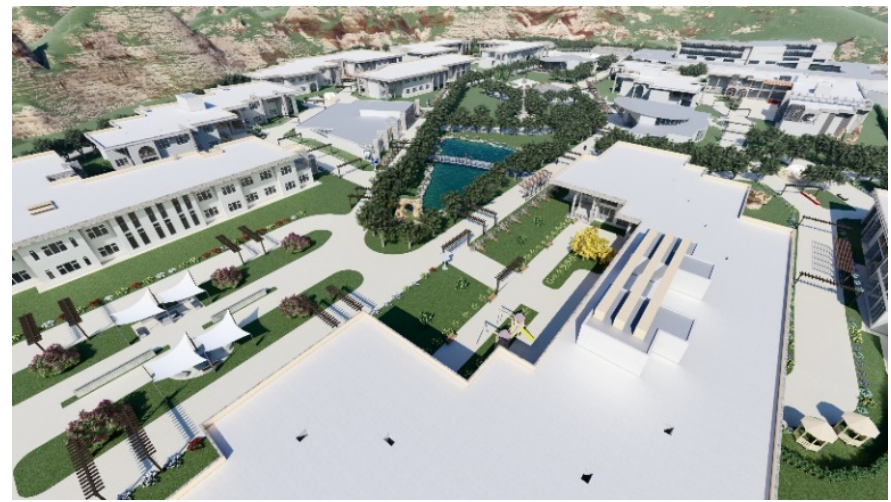

A-Perspective of the proposed project
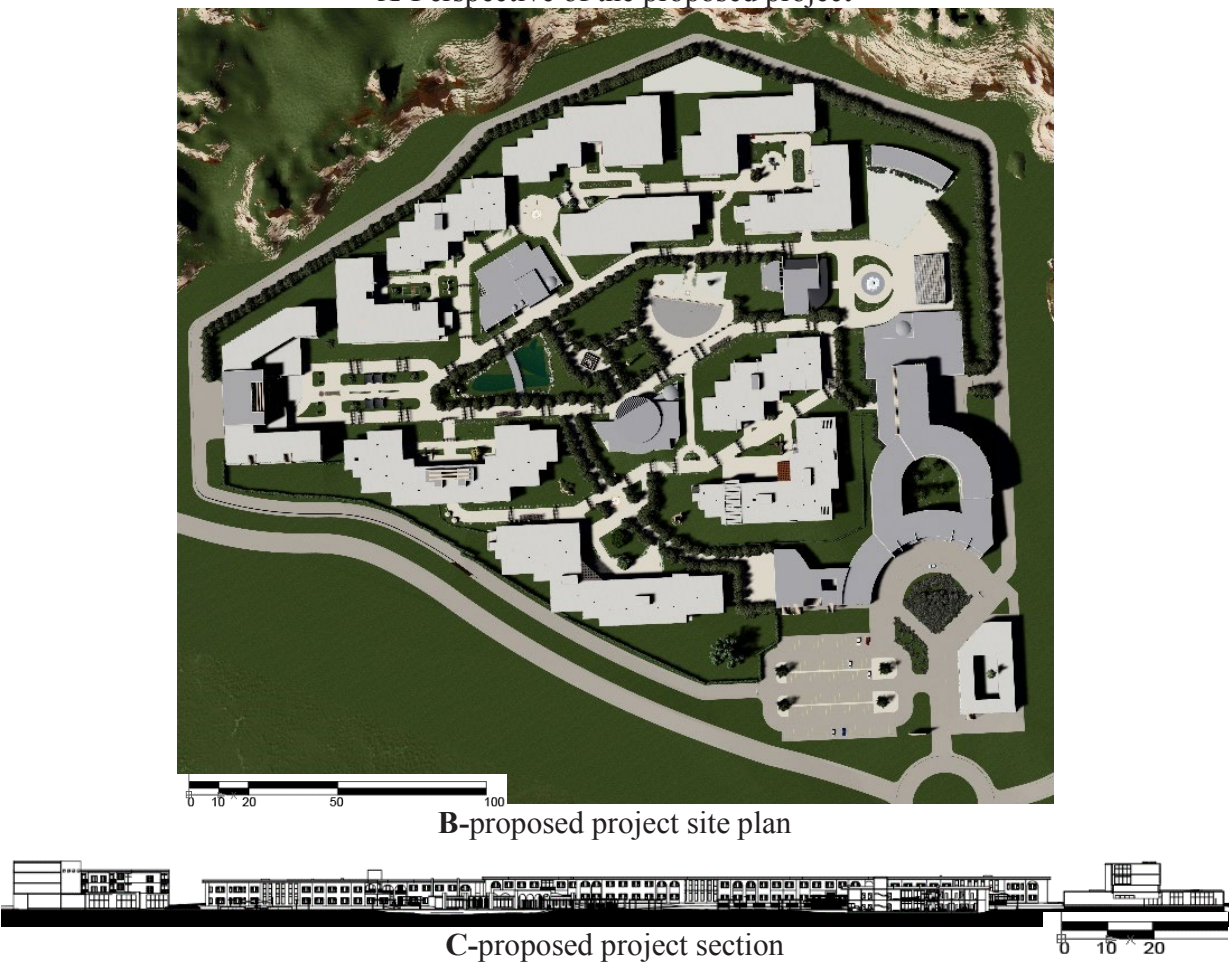

Fig. 1. Proposed project designed by the researcher 2020 (A, B, C).

\section{Conclusion}

This narrative review investigated the design of pathways that are appropriate and compatible with the psychological and physiological needs of Alzheimer's patients. Although many scientists and clinicians have increasingly studied the relationship between outdoor landscapes and health and the healing process, there is insufficient evidence to support the use of the outdoor natural landscape for Alzheimer's patients.

However, a large amount of research has demonstrated the therapeutic potential of outdoor landscapes on individuals' health and well-being. In addition to these studies, focused on environmental conditions and conditions similar to those of Alzheimer's 
patients, which in the case that they cannot meet the needs of patients, they can at least transform the environments into more suitable places to live.

Based on the analysis of these studies, a set of recommendations were concluded. The most important of them are:

1. The presence of contrast in the visual shapes helps to enhance visual memory, whether in terms of texture or color.

2. The presence of distinct smells or sounds in some places can trigger spatial memory and help patients feel familiar in the areas that are frequently visited

3. Clear, easy-to-remember entrances with distinct attractive elements are to help patients find their target.

4. It is important to hide the exits in a way that prevents the patients from searching for them rather than only preventing these patients from leaving.

5. The presence of the natural environment and water elements helps the healing process and slows down the speed of deterioration of the patient's condition.

6. Attention must be paid to the width of the pathways, as the patients often do not walk individually, and the change of width of the pathways in some areas may give patients greater freedom of movement.

7. The presence of steep slopes is not desirable as we are dealing with the elderly in the first place.

8. Shading of the paths is important, especially in warm areas, but it must be noted that Alzheimer's patients suffer from a kind of anxiety due to the effect of sharp shadow forming on the ground. So using coverage that generates a kind of gradient shade can alleviate this anxiety.

9. Having slow-changing scenes helps reduce confusion.

10. Reducing the number of intersections in the paths helps to reduce options for an Alzheimer's patient, thus facilitating the decision-making process.

\section{References}

1. World Population Ageing 2017. Un. org. N. p., (2019), https:/www.un.org/en/development/desa/population/theme/ageing/WPA2017.asp.

2. R. Peters, The prevention of dementia, Geriatric Psychiatry, 24, 452-458 (2009), DOI: 10.1002/gps.2153

3. M. Prince, Dementia in developing countries. A consensus statement from the 10/66 Dementia Research Group, Geriatric Psychiatry, 15, 14-20, (2000), DOI: 10.1002/(SICI)1099-1166(200001)15:1<14::AID-GPS70>3.0.CO;2-8

4. J. A. Kaufman, Nature, Mind, and Medicine: A Model for Mind-Body Healing, Explore, 14, 268-276, (2018), DOI: 10.1016/j.explore.2018.01.001

5. Age-Friendly Environments. World Health Organization. N. p., 2018. https://www.who.int/ageing/projects/age-friendly-environments/en/

6. R. Gorman, Smelling therapeutic landscapes: Embodied encounters within spaces of care farming, Health \& Place, 47, 22-28, (2017), DOI: 10.1016/j.healthplace.2017.06.005

7. J. T. Mokos, Stigmatized places as therapeutic landscapes, Medicine Anthropology Theory, 4, 123-151, (2020), DOI: 10.17157/mat.4.1.362

8. D. Nutsford, A. L. Pearson, S. Kingham, F. Reitsma, Residential exposure to visible blue space (but not green space) associated with lower psychological distress in a capital city, Health \& Place, 39, 70-78, (2016), DOI: 10.1016/j.healthplace.2016.03.002 
9. S. Dempsey, M. T. Devine, T. Gillespie, S. Lyons, A. Nolan, Coastal blue space and depression in older adults, Health \& Place, 54, 110-117, (2018), DOI: 10.1016/j.healthplace.2018.09.002

10. T. Sulander, E. Karvinen, M. Holopainen, Urban Green Space Visits and Mortality Among Older Adults, Epidemiology, 27, 34-35, (2016), DOI: 10.1016/j.healthplace.2018.09.002

11. W. Nagib, A. Williams, Creating «therapeutic landscapes» at home: The experiences of families of children with autism, Health \& Place, 52, 46-54, (2018), DOI: 10.1016/j.healthplace.2018.05.001

12. J. M. Finlay, 'Walk like a penguin': Older Minnesotans' experiences of (non)therapeutic white space, Social Science \& Medicine, 198, 77-84, DOI: 10.1016/j.socscimed.2017.12.024

13. S. L. Bell, R. Foley, F. Houghton, A. Maddrell, A. M. Williams, From therapeutic landscapes to healthy spaces, places and practices: A scoping review, Social Science \& Medicine, 196, 123-130, (2018), DOI: 10.1016/j.socscimed.2017.11.035

14. M. Handley, F. Bunn, C. Goodman, Dementia-friendly interventions to improve the care of people living with dementia admitted to hospitals: a realist review, BMJ Open, 7, (2019), DOI: 10.1016/j.maturitas.2019.06.011.

15. G. Hansen, E. Alvarez, Landscape Design: Analyzing Site Conditions, ENH1165, (2016), URL: http://edis.ifas.ufl.edu/ep426

16. G. D. S. Ludden, T.J.L. van Rompay, K. Niedderer, I. Tournier, Environmental design for dementia care - towards more meaningful experiences through design, Maturitas, 128, 10-16, (2019), DOI: 10.1016/j.maturitas.2019.06.011

17. M. Handley, F. Bunn, C. Goodman, Dementia-friendly interventions to improve the care of people living with dementia admitted to hospitals: a realist review, BMJ Open, 7, (2019), DOI: 10.1016/j.maturitas.2019.06.011

18. Kevin Lynch, The Image of the City (Cambridge, Mass: MIT Press, 1960)

19. Kevin Lynch, Site Planning (Cambridge: M.I.T. Press, 1971)

20. J. McCluskey, Road form and townscape (Architectural Press London, 112-132, 1979)

21. J. O. Simonds, B.W. Starke, Landscape Architecture: A Manual of Environmental Planning and Design, New York: McGraw-Hill, (2006)

22. Y. Ashihara, Exterior Design in Architecture. New York: Van Nostrand Reinhold, 1970. Internet resource.

23. P. Motealleh, W. Moyle, C. Jones, K. Dupre, Creating a dementia friendly environment through the use of outdoor natural landscape design intervention in long-term care facilities: A narrative review, Health \& Place, 58, (2019), DOI: 10.1016/j.healthplace.2019.102148

24. N. Hendriks, L. Huybrechts, K. Slegers, A. Wilkinson, Valuing implicit decisionmaking in participatory design: A relational approach in design with people with dementia, Design Studies, 59, 58-76, DOI: 10.1016/j.destud.2018.06.001

25. P. C. Uwajeh, T. O. Iyendo, M. Polay, Therapeutic gardens as a design approach for optimizing the healing environment of patients with Alzheimer's disease and other dementias: A narrative review, Explore, 15, 352-362, (2019), DOI: 10.1016/j.explore.2019.05.002

26. D. Kreps, O. Burmeister, I am a Person: How value sensitive design can better support caring relationships for people with dementia, interpreted through the lens of personhood, The Orbit Journal, 2, 1-12, (2019), DOI: 10.29297/orbit.v2i2.79

27. D. Wei, X. Li, Measuring the spatial quality of bedrooms in nursing homes with visual environmental performance, Frontiers of Architectural Research, (2021), DOI: 10.1016/j.foar.2021.01.003 
28. A. M. Minibaeva, E. V. Denisenko, Background and trends in the development of architecture of homes for the elderly in domestic and foreign design experience, Izvestiya KGASU, 48, 64-72, (2019) 